\title{
Management and control of asthma as assessed by actual/best function and corticosteroid use 1980-1993/4
}

\author{
C.K. Connolly*, S.M. Alcock+, R.J. Prescott
}

Management and control of asthma as assessed by actual/best function and corticosteroid use 1980-1993/4. C.K. Connolly, S.M. Alcock, R.J. Prescott. CERS Journals Ltd 1998. ABSTRACT: This study aimed to determine whether the changes in practice in the management of asthma since the early 1980s have improved standards (as assessed by higher actual/best function) and reduced the need for oral corticosteroids.

All asthmatic outpatients were reviewed in 1980, 1983, 1988/89 and 1993/94. Therapeutic step, defined by suppressive medication alone, actual and best peak expiratory flow (PEF) were recorded. Cohorts from 1980, 1983 and 1988/89 were identified in whom best function was established on all subsequent occasions. Changes in practice demonstrated by cross-sectional review of all subjects were interpreted with the aid of longitudinal analysis of the cohorts.

Attendance increased from 463 in 1980 to 772 in 1993/94. Between 1983 and 1993/ 94, the proportion maintained on inhaled corticosteroids increased from 49 to $84 \%$ with increased use of higher doses. Mean actual/best PEF rose from 80 to $87 \%$, improving at each therapeutic step. The proportion needing rescue oral corticosteroids fell from 47 to $35 \%$ and maintenance oral corticosteroids from 20 to $9 \%$. In the cohorts, there was a similar reduction in use of rescue corticosteroids, but not of maintenance oral corticosteroids.

The study confirmed an increase in the use and dose of inhaled corticosteroids, and a better outcome at all treatment steps. The fall in the proportion of subjects dependent on oral corticosteroids was due to attrition, rather than weaning in later years. Eur Respir J 1998; 12: 859-864.
*Dept of Medicine, University of Newcastle upon Tyne, Newcastle upon Tyne, UK +Dept of Medicine, Darlington Memorial Hospital NHS Trust, Darlington, UK. Medical Statistics Unit, University of Edinburgh, Edinburgh, UK

Correspondence: C.K. Connolly, Darlington Memorial Hospital, Hollyhurst Road, Darlington, Co. Durham DL3 6HX, UK. Fax: 441325743435

Keywords: Actual/best function asthma

best function

inhaled corticosteroids

oral corticosteroids

therapeutic step

Received: August 191997

Accepted after revision May 211998

Supported financially by the National Asthma Campaign, GlaxoWellcome and Breathe North.
The management of chronic asthma lends itself to a stepwise approach, which has been developed in national and international guidelines over the past 10 yrs $[1,2]$. Potentially preventive treatment, including oral corticosteroids, first used in the $1950 \mathrm{~s}$, cromoglycate from the 1960s [3] and inhaled corticosteroids since the 1970s [4], have been available for a much longer period, but it was not until the 1980s that the use of inhaled corticosteroids became universally accepted as the best management for the majority of patients with persistent symptoms. During this decade, the range of available preparations was extended in terms of both dose and delivery systems. In addition, advice with regard to environmental control has been clarified, the level of education has increased [5] and patient expectations have increased.

Actual over best or maximum function (actual/best) is accepted as a spot measure of control. It was first described in the context of a stepwise approach to management of asthma in 1983 [6, 7]. It has now been validated [8] and incorporated into guidelines [9]. A publication in 1983 [6] described an audit exercise in 1980. Following this, a long-term study of asthma was started in 1983 [10, 11], with further entry and review in 1988/89 and 1993/ 94. On all four occasions, best and actual/best peak expiratory flow (PEF) of all patients was recorded, together with their current treatment.

This study looked at the management of subjects who attended on each occasion in relation to cohorts of sub- jects who attended at all subsequent times from 1980, 1983 and 1988, respectively. These cohorts, who excluded patients dying during the interval, represent a survivor population, and so differ in some respects from the clinical population as a whole. Nevertheless, being subject to analysis each year, the cohorts provide a control group to confirm apparent secular changes deduced from the crosssectional analyses of the whole clinic population, which might vary in severity or other characteristics from year to year. It was also accepted that improvements in outcome might be due to other factors such as education and that increases in therapy might reflect unsuccessful attempts at satisfying increased expectations as well as real improvements in management. Nevertheless, this study aimed to assess whether there was any improvement in standards assessed by the actual/best PEF achieved overall and at each therapeutic step and to see whether there is any reduction in exacerbations requiring the use of rescue oral corticosteroids.

\section{Methods}

\section{Subjects}

All patients attending one of the author's (CKC) consultant clinics (including privately) with a clinical diagnosis of asthma, supported by the demonstration of reversibility of peak flow rate by $\breve{S} 15 \%$ to $\breve{S} 200 \mathrm{~L} \cdot \mathrm{min}^{-1}$, were eligible. 
This included home recordings of PEF in the majority of subjects. When the clinical diagnosis was in doubt it was endorsed by the demonstration of sputum or blood eosinophilia. Subjects treated with oral corticosteroids primarily for a diagnosis other than asthma were excluded from this study.

\section{Entry and review}

Subjects were entered into the study and reviewed as follows:

1) all subjects attending the author's clinics, 1980;

2) all subjects attending the author's clinics for at least $1 \mathrm{yr}$ at entry and who were expected to stay in the district (database for Darlington and Northallerton long-term asthma study), 1983;

3) first review in the long-term study, 1988/89, with new entrants referred between 1983 and 1988; and

4) second review in the long-term study, 1993/94, with new entrants referred between 1988 and 1993.

Entry in 1980 and 1983 for the calendar year, and in 1988/89 and 1993/94 from 1 April to 31 March. In 1988/ 99 and 1993/94, subjects were divided into those who were attending routinely (clinic attenders) and those who were recalled especially for the study (special attenders).

\section{Groups for follow-up}

Cohorts for longitudinal analysis. Those subjects who first attended in the relevant year and had best function assessed on all subsequent occasions including 1993/94 comprised the 1980, 1983 and 1988 cohorts.

Groups for cross-sectional analysis. The results for each year are presented as appropriate for either all clinic attenders or all those seen (all attenders), i.e. clinic attenders plus special attenders.

\section{Regimen status}

Therapeutic regimen was accepted as stable if it was mutually satisfactory to both patient and clinician and had not been altered for 3 months. Patients who were not satisfactory were reviewed and re-recorded as stable if a satisfactory regimen had been maintained within 3 months of the close of entry. The study was planned before the publication of consensus guidelines for the management of asthma. Although a stepwise approach has been used throughout, bronchodilators were not used in the definition of the higher steps. As far as the databases allow it, the following steps are used: A: bronchodilator only; B: disodium cromoglycate (DSG)/nedocromil; $\mathrm{C} 1$ : inhaled corticosteroids $<800 \mu \mathrm{g}$; C2: inhaled corticosteroids 800-1000 $\mu \mathrm{g}$; C3: inhaled corticosteroids $>1000 \mu \mathrm{g}$; D1: oral steroids < $10 \mathrm{mg}$; D2: oral steroids Š10 mg; and U: unstable.

\section{Pulmonary function}

Actual PEF was recorded at the index visit. If satisfactory control was only achieved later in the observation period, actual/PEF was amended to that at the relevant visit.

In 1980, best function was the best recorded PEF after bronchodilator, ignoring obvious "rogue" readings. For subsequent years, best function was the best recorded in the notes from 1 January of the previous year, using the following protocol [10]: $>80 \%$ predicted, after bronchodilator; 70-80\% predicted, after bronchodilator, on satisfactory preventive treatment, with PEF recording at least twice daily for 5 days; $<70 \%$ predicted, after bronchodilator, on $30 \mathrm{mg}$ prednisolone for at least 1 week, with consistent readings over the last $48 \mathrm{~h}$. Best PEF is expressed as per cent predicted [12] and actual/best as a simple percentage.

\section{Ethics approval}

The study was approved by the Darlington and Northallerton Ethics Committees.

\section{Statistical methods}

The presentation is largely descriptive. Group means were compared using the independent sample t-test or paired t-test as appropriate and proportions were compared with the Chi-squared test. All calculations were performed using Version 6.04 of SAS of SAS for the PC (SAS Institute Inc., Cary, NC, USA). Discrepancies in some totals are due to occasional missing values.

\section{Results}

\section{General}

The details of the subjects are shown in table 1 . The mean values are unaltered by the exclusion of special attenders recalled for the study. The cohort subjects were similar to survivors with incomplete records recruited in the same year, but were younger and had a shorter duration of asthma than those who died. Otherwise, the only major difference was a shortfall of males in the 1980 cohort (38\% versus $47 \%$ all survivors). The 1980, 1983 and 1988 cohorts comprised approximately $43 \%, 60 \%$ and $95 \%$ of their respective survivors.

\section{Distribution of regimen}

Clinic attenders (cross-sectional analysis). The crosssectional distribution of regimen for clinic attenders in each year is shown in table 2 , reflecting current clinic policy. The proportion on bronchodilator alone was higher in

Table 1. - Demographic details of subjects showing the three cohorts and all attenders in each year

\begin{tabular}{lrrrrrrrr}
\hline & \multicolumn{3}{c}{ Cohorts } & & \multicolumn{4}{c}{ All attenders } \\
\cline { 2 - 6 } \cline { 6 - 8 } & 1980 & 1983 & 1988 & & 1980 & 1983 & $1988 / 89$ & $1993 / 94$ \\
\hline $\mathrm{n}$ & 133 & 193 & 251 & & 463 & 624 & 733 & 772 \\
Male \% & 36 & 51 & 53 & 47 & 49 & 50 & 50 \\
Mean age yrs & 48 & 49 & 49 & 50 & 52 & 54 & 56 \\
Mean duration yrs & 16 & 17 & 15 & & - & 19 & 19 & 22 \\
Never-smokers \% & 57 & 45 & 50 & 48 & 47 & 49 & 43 \\
\hline
\end{tabular}


Table 2. - Distribution of regimen in clinic attenders 1980-1993/94

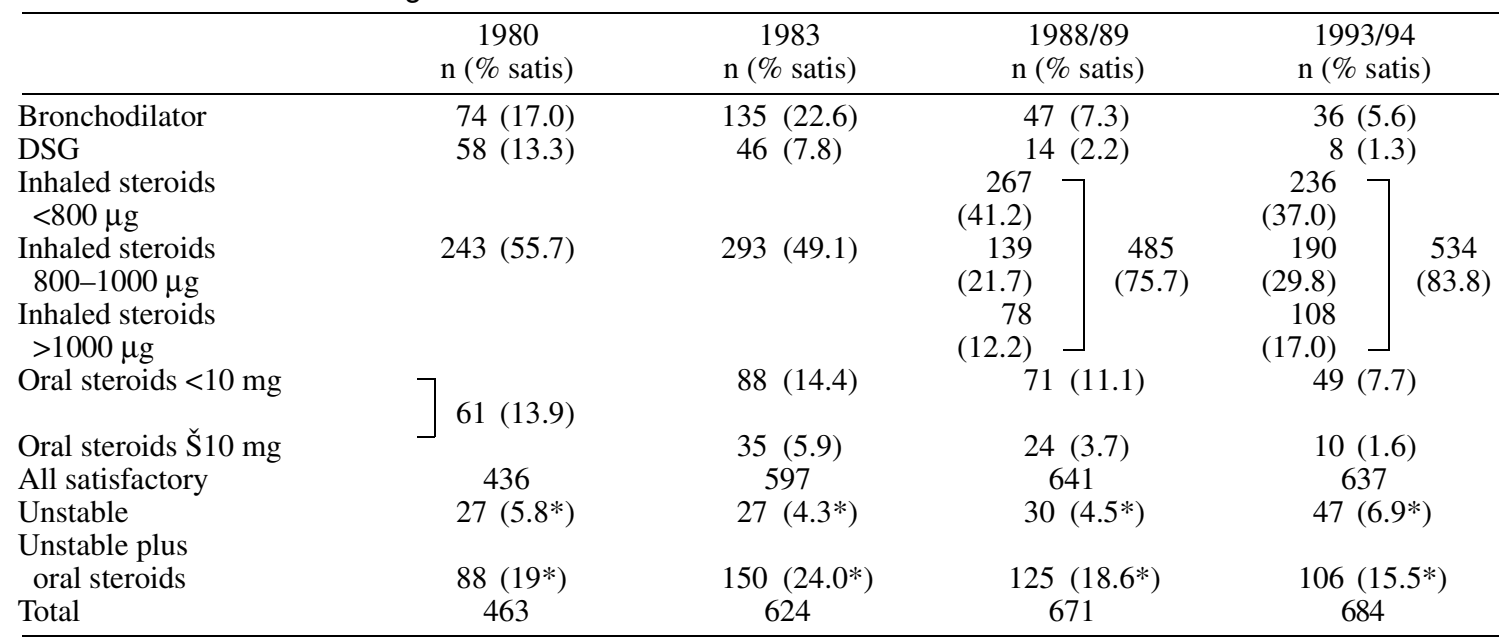

DSG: disodium cromoglycate; satis: satisfactory. *: percentage of total.

1983 than 1980, but fell thereafter. Management with cromoglycate alone fell consistently. After 1983 the proportion on inhaled steroids increased, with fewer requiring oral corticosteroids. The dose of inhaled steroids increased between 1988/89 and 1993/94, as did the proportion recorded as unstable (4.5\% versus $6.9 \%)$. The latter was offset by a fall in the proportion of patients on oral corticosteroids from $14.8 \%$ in $1988 / 89$ to $9.3 \%$ in $1993 /$ 94. The use of rescue oral steroids fell from $47 \%$ in 1983 to $33 \%$ in $1989(\mathrm{p}<0.01)$. There was little change between 1988/89 and 1993/94 (table 3).

Cohorts (longitudinal analysis). As the cohorts included specially recalled subjects, the appropriate comparator group is all attenders. The inclusion of special attenders recalled for the study increased the proportion of subjects on bronchodilator only and unstable by $<1 \%$, as compared with regular clinic attenders. The distribution of the main regimen step is shown for these subjects and each of the cohorts in table 2 . There was a progressive increase in the use of inhaled corticosteroids. Most of the increase took place during the first two intervals, 1980-1983 and 1983$1988 / 89$. There was a fall in the numbers unstable in the first interval, in those on bronchodilators only in the second, and on DSG throughout. Between 1983 and 1988/89 the changes in the distribution of therapy were similar in the 1980 and 1983 cohorts, with no further change in any of the three cohorts in the last period. In contrast, the proportion of all attenders on inhaled steroids increased by $24.7 \%$ between 1983 and 1988/89 and a further $8.6 \%$ in the last period. Although the numbers on inhaled corticosteroids did not change between 1988/89 and 1993/94, the proportion on higher doses increased in all groups except for the 1988 cohort.

The proportion of all attenders on oral corticosteroids fell, but there was little change in any of the cohorts. Ten subjects of the 1980 cohorts were stable on oral corticosteroids in 1993/94. Seven of these were on oral steroids at each occasion, although two were unstable in 1993/94. The experience in the other cohorts was very similar. Whilst the use of regular oral corticosteroids remained consistent, the numbers requiring booster courses fell in all relevant groups between 1983 and 1989, but with little change thereafter (table 3).

\section{Relationship between regimen and pulmonary function}

Best peak flow. As the formal protocol for best function was not in use in 1980, 1983 is the starting year for figure 1, which shows best PEF of clinic attenders at each principal treatment step. The level at each step shows little increase with time, with the exception of those on bronchodilator only. However, when inhaled corticosteroids

Table 3. - Percentage of subjects on inhaled corticosteroids taking one or more booster courses of oral steroids in the previous year

\begin{tabular}{|c|c|c|c|c|c|}
\hline \multirow[b]{2}{*}{ Year } & \multicolumn{3}{|c|}{ Cohort } & \multicolumn{2}{|c|}{ Attenders } \\
\hline & 1980 & 1983 & 1988 & Clinic & All \\
\hline 1983 & 41 & 50 & - & \multicolumn{2}{|c|}{47} \\
\hline 1988/89 & 34 & $33 * *$ & 35 & $33 * * *$ & $29 * * *$ \\
\hline Low dose $e^{\dagger}$ & 29 & 19 & 20 & 23 & 22 \\
\hline Intermediate dose & 34 & 28 & 42 & 39 & 38 \\
\hline High dose $\#$ & 47 & 66 & 66 & 61 & 60 \\
\hline 1993/94 & 34 & 36 & 38 & 39 & 35 \\
\hline Low dose $\dagger$ & 31 & 22 & 25 & 23 & 24 \\
\hline Intermediate dose & 35 & 33 & 42 & 39 & 39 \\
\hline High dose \# & 78 & 66 & 71 & 75 & 74 \\
\hline
\end{tabular}

: $<800 \mu \mathrm{g}$; : 800-1000 $\mu \mathrm{g}$; \#: >1000 $\mu \mathrm{g}$ inhaled steroids. **: $\mathrm{p}<0.01 ; * * *: \mathrm{p}<0.001$ for $1988 / 89$ versus 1983. 


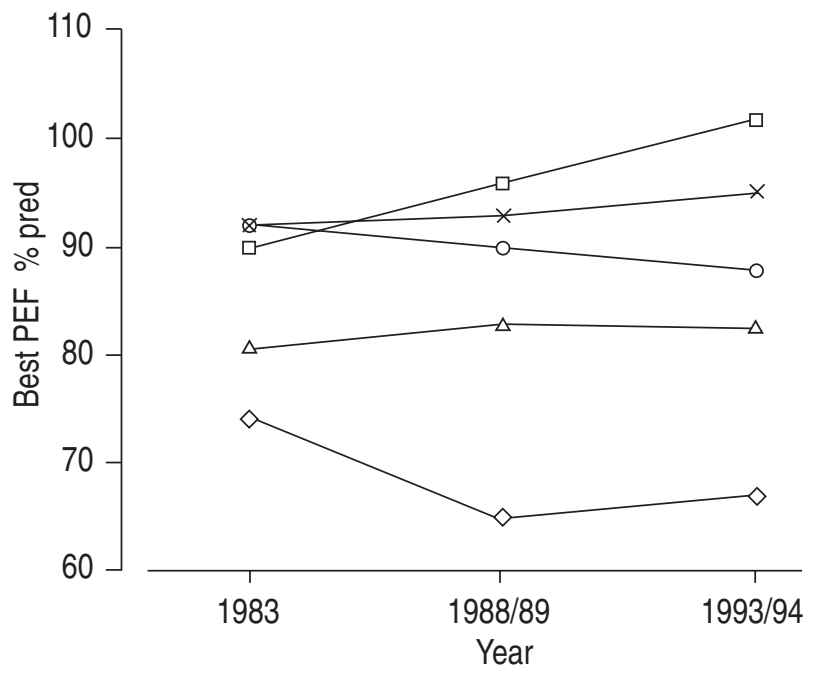

Fig. 1. - Best peak expiratory flow (PEF) of clinic attenders at each main treatment step from 1983 to 1993/94. $\square$ : Bronchodilators only; ○: DSG; $\times$ : inhaled steroids; ý: oral steroids $<10 \mathrm{mg} ; \diamond$ : oral steroids Š10 $\mathrm{mg}$.

are stratified by dose (fig. 2), there was a clear separation and the best PEF of those on low-dose inhaled steroids increased from $97 \%$ in $1988 / 89$ to $103 \%$ in $1993 / 94$ ( $\mathrm{p}<$ $0.001)$.

Actual/best peak flow. There was a small fall in mean actual/best PEF of clinic attenders between 1980 and 1983. This was probably associated with less rigorous assessment of best PEF in 1980. Thereafter, actual/best PEF improved, with the greatest difference between 1983 and 1988/89 but an improvement was demonstrated for each treatment step for both of the intervals (1983-1988/89 and 1988/89-1993/94) (fig. 3). The results for the 1980 and 1983 cohorts were similar and virtually identical to each other. The improvement in actual/best function was mainly between 1983 and 1988/89.

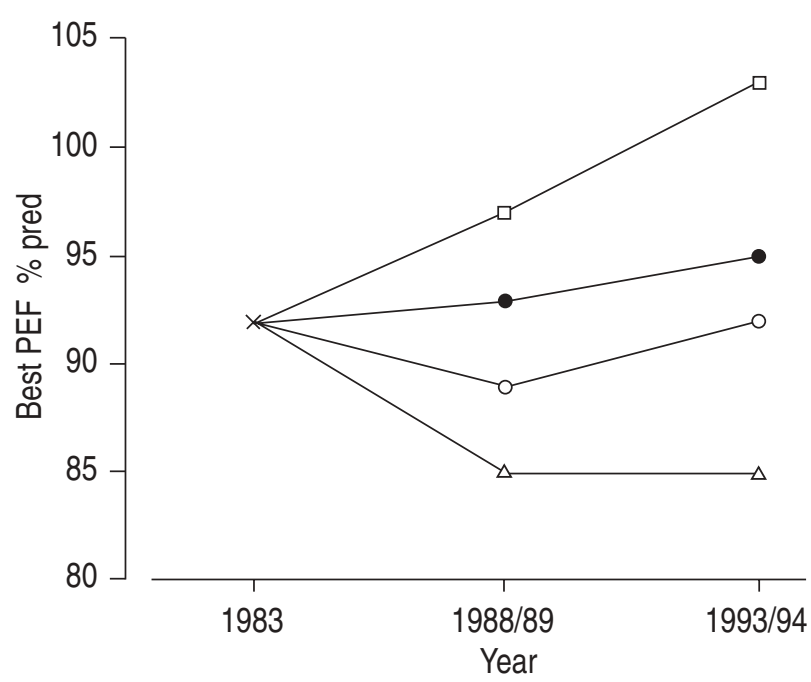

Fig. 2. - Best peak expiratory flow (PEF) for patients on the inhaled corticosteroid steps from 1983 to 1993/94. The values for those on lowdose $(<800 \mu \mathrm{g} ; \square)$, intermediate-dose $(800-1000 \mu \mathrm{g} ; \bigcirc)$ and high-dose $(>1000 \mu \mathrm{g} ;$ ý) inhaled steroids are shown for 1988/89 and 1993/94. X: all; $\bullet$ : mean overall.

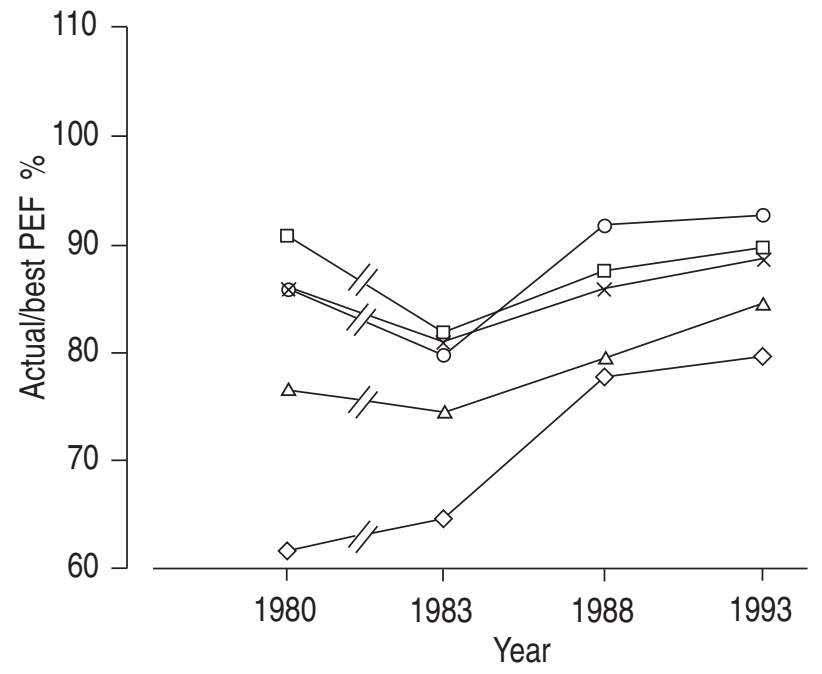

Fig. 3. - Actual/best peak expiratory flow (PEF) at each main treatment step from 1980 to 1993/94. (The lines between 1980 and 1983 are broken because the criteria for best function were less strict in 1980.) : Bronchodilators only; $\bigcirc$ : DSG; $\times$ : inhaled steroids; ý: oral steroids $<10$ $\mathrm{mg} ; \diamond$ : oral steroids Š $10 \mathrm{mg}$.

The mean actual/best PEF of unstable subjects increased from $76 \%$ in 1983 to $83 \%$ in $1988 / 89$ ( $\mathrm{p}<0.05)$ in clinic attenders as a whole but not in the two cohorts, where the change was insignificant $(80 \%$ versus $82 \%$, $\mathrm{p}>0.1)$. There was little change in the actual/best function in any group during the period 1988/89-1993/94.

\section{Outcome and entry regimen}

The outcome of 251 subjects who were stable without requiring oral corticosteroids in 1983 and who were seen in 1983 and 1993, is summarized in table 4. Group A were not on inhaled corticosteroids at entry and group B were managed with inhaled corticosteroids in 1983. On stratification by sex, best PEF on study entry was similar in the two groups. The small and nonsignificant loss of best function over the $10 \mathrm{yrs}$ was virtually identical. The average therapeutic step in 1993/94 approximated to a median dose of $600 \mu \mathrm{g}$ of inhaled corticosteroids in group A and $900 \mu \mathrm{g}$ in group B. The findings between 1980 and 1993 in the 127 stable subjects seen in both years, but not requiring oral corticosteroids in 1980, were very similar.

Table 4. - Best peak expiratory flow (PEF) in 1983 and 1993 of subjects not using (group A) and using (group B) inhaled corticosteroids in 1983 (unstable patients and those on oral corticosteroids in 1983 are excluded)

\begin{tabular}{|c|c|c|c|c|}
\hline & \multicolumn{2}{|c|}{$\begin{array}{c}\text { Males } \\
\text { Maximum } \\
\text { PEF L·min }{ }^{-1}(\mathrm{SD})\end{array}$} & \multicolumn{2}{|c|}{$\begin{array}{c}\text { Females } \\
\text { Maximum } \\
\text { PEF L·min }{ }^{-1}(\mathrm{SD})\end{array}$} \\
\hline & Group A & Group B & Group A & Group B \\
\hline & 40 & 76 & 46 & 95 \\
\hline 1983 & $456(120)$ & 486 (104) & 354 (66) & $373(83)$ \\
\hline 1993 & $420(120)$ & 453 (106) & 350 (95) & $354(84)$ \\
\hline Change & $-36 \quad(52)$ & $-32(64)$ & $-4(74)$ & $-19(53)$ \\
\hline
\end{tabular}

Differences between groups A and group B were nonsignificant in all cases. 


\section{Discussion}

The object of this study was to describe changes in clinic practice as reflected in the distribution of therapeutic steps and to seek associated improvement in standards. It compared performance on four occasions over a 14yr period and was therefore, essentially, a cross-sectional analysis. The purpose of the cohorts was to control for possible different characteristics of new entrants on each occasion. Improved standards may arise from improved environmental control, improved education and higher expectations of patients, as well as from improved drug therapy. Indeed, increased intensity of therapy might reflect patients' and physicians' expectations of better control without actually achieving the expected improvement. This study attempted to analyse whether there has been any improvement in standards and outcome, as judged by actual/best function, exacerbations requiring oral steroids and the proportion of patients on a stable regimen. It was appreciated that if an improvement in outcome were demonstrated in association with an increase in therapy, other factors may well have contributed.

The use of actual/best function in assessing response may be criticized on the grounds that a single measurement is unlikely to be helpful in such a variable condition as asthma. However, it has the advantage that actual PEF is a simple, routine measure which should be made at most if not all clinics. Whilst a simple reading may not be as sensitive a functional measurement of control as change in $\mathrm{PEF}$, it is frequently used in clinical practice. Furthermore, in addition to assessing the patient's stage, it is a measure of what the clinician regards as acceptable. It has been shown that it is possible to achieve consistent actual/best PEF in groups of patients on different therapeutic regimes. It is accepted as an appropriate outcome measure $[12,13]$ and incorporated in current British guidelines [9]. It is a reasonable assumption that higher levels of actual/best PEF at the same therapeutic step may reflect a more aggressive approach on the part of the clinician, as well as better symptomatic control. Furthermore, despite the disadvantage of a spot measurement, symptoms are related to actual/best function in this group of patients [14].

Actual/best PEF also depends on accurate assessment of the denominator. The criteria for the establishment of best function were not as rigorous in 1980 as those required by the protocol in subsequent years. Therefore, the results in 1980 are not strictly comparable with the later ones. From 1983 the majority of subjects satisfied the protocol [10], but those who did not were excluded from the analysis of actual/best function, because the study was attempting to assess clinical practice. The clinician can only make use of the physiological information available at the time of his or her therapeutic decision.

After 1983, actual/best PEF improved consistently, not only in the clinics as a whole, but also in the 1980 and 1983 cohorts. This suggests that standards were indeed raised, an interpretation supported by the reduction in the proportion of patients requiring rescue oral corticosteroids. The increase in actual/best PEF was consistent across the therapeutic steps. Between 1983 and 1988/89, when this change was at its greatest in those on satisfactory treatment, the actual/best PEF of those deemed unstable increased in parallel. This confirms that the improvement reflected a more aggressive approach and was not an arte- fact arising from improved categorization of the subjects. The best function of all patients on inhaled corticosteroids only increased by $3 \%$ in the 10 yrs between 1983 and 1993/94, but appropriate selection of inhaled steroid dose is suggested by the inverse relationship between best function and dose, which was present in 1988/89, and stronger in 1993/94.

In the cross-sectional analyses, new recruitment reduces the survivor effect, which necessarily affects results derived from the cohort which excluded those dying during the observation period. In an analysis of outcome, mortality during the period 1983-1993/94 was inversely related to best FVC and PEF on study entry [15]. Subjects on oral steroids tended to have poor best function on entry and, therefore, the attrition rate of those starting on this therapeutic step was high. In all three cohorts of survivors the proportion of subjects on oral steroids did not alter, despite the progressive fall in the clinics as a whole. This suggests that the proportion of those recruited recently who required oral steroids was greatly reduced.

In recent years, general practitioners have been encouraged to be more selective in their referrals, which should increase rather than decrease the proportion of patients requiring more intense therapy. This is unlikely to be fully counterbalanced by milder patients being referred because of increased patient demand or increased interest in occupational asthma. Differences in referral policy are, therefore, unlikely to explain the progressive reduction in the proportion of new recruits requiring oral corticosteroids and the possibility that this is related to the early introduction of inhaled corticosteroids before referral must be considered. The analysis of entry step and outcome presented here does not support this hypothesis, but in this respect the numbers are small and appropriate selection of subjects for the early introduction of inhaled corticosteroids may have confounded the issue. Despite these results, the contention of AGERTOFT and PEDERSON [16] that the early introduction of inhaled corticosteroids is important in chil-dren may also apply to adults. Pulmonary function and response to treatment are inversely related to duration of asthma $[10,11]$ and early studies suggest that better outcome might be achieved with earlier treatment with inhaled corticosteroids $[16,17]$, but further work is required to confirm this.

Despite the fall in numbers on oral corticosteroids, the proportion of patients whose therapy was not classed as mutually satisfactory rose between 1983 and 1993/94. In 1980 and 1983, inhaled corticosteroids were almost always prescribed in what is now the standard low dose $(400 \mu \mathrm{g}$. day $^{-1}$ ). The measurements from those years are compatible with a target actual/best PEF of $80 \%$, rather than $85 \%$, which is the current clinic practice. The increase in range of dose, preparation and delivery device allows more scope to tune therapy to satisfy the higher expectations of patient and clinicians. If the target is raised, then patients who have been regarded as satisfactory in the earlier years might now be classified as unstable. Thus, an increase in the proportion of patients recorded as unstable does not necessarily mean a lowering of either standards or outcome.

In conclusion, there has been an improvement in standards, as assessed by actual/best function and reflected in the reduction in the need for rescue courses of oral corticosteroids. This is probably associated with greater expectations, the more appropriate use of prophylactic medication 
and, possibly, other nondrug interventions throughout the course of the disease. In contrast, the reduction in the proportion of patients on oral corticosteroids in later years could not be explained by changes in hospital practice, but may reflect better early management in primary care.

\begin{abstract}
Acknowledgements: The authors acknowledge the contribution made to the study by M. Mamun, present research fellow, N.K. Murthy, A.A. Gatnash and P.M. Roy, former research fellows, T.J. Harding and J.S. Jackson, research assistants.
\end{abstract}

\section{References}

1. British Thoracic Society, Research Unit of the Royal College of Physicians of London, King's Fund Centre, National Asthma Campaign. Guidelines for management of asthma in adults: I - chronic persistent asthma. BMJ 1990; 301: 651-654.

2. Sheffer AL, Chairman. International consensus report on diagnosis and management of asthma. Clin Exp Allergy 1992; 22: Suppl. 1.

3. Moran F, Bankier JDH, Boyd G. Disodium cromoglycate and the treatment of allergic bronchial asthma. Lancet 1968; ii: 137-139.

4. Cameron SJ, Cooper EJ, Crompton GK, Hoare MV, Grant IWB. Substitution of beclomethasone dipropionate aerosol for oral prednisolone in the treatment of chronic asthma. BMJ 1973; 4: 205-207.

5. Partridge MR. Delivering optimal care to the person with asthma, what are the key components and what do we mean by patient education. Eur Respir J 1995; 8: 298305.
6. Connolly CK. Management of asthma in outpatients. $J R$ Coll Phy Lon 1983; 17: 115-120.

7. Connolly CK. Age and the prescription of drugs for asthma. Br J Clin Pract 1984; 38: 45-48.

8. Connolly CK, Prescott RJ, Alcock SM, Gatnash AA. Actual over best function as an outcome measure for asthma. Respir Med 1994; 88: 453-459.

9. British Thoracic Society, et al. (Woodhead M, ed). Guidelines on the management of asthma. Thorax 1995; 52: Suppl. 1.

10. Connolly CK, Chan NS, Prescott RJ. The relationship between age and duration of asthma and the presence of persistent obstruction in asthma. Post Grad Med J 1988; 64: 422-425.

11. Connolly CK, Prescott RJ. Pulmonary function and drug regimens in asthmatics. Br J Clin Pract 1990; 44: 148153.

12. Cotes JE. Lung Function, 4th Edn. Oxford, Blackwell Scientific Publications, 1979.

13. Bucknall CE. Definitions of severity and outcome measures. Respir Med 1996; 90: 447-452.

14. Alcock SM, Mamun M, Prescott RJ, Connolly CK. Symptoms and pulmonary function in asthma. Respir Med 1998; in press.

15. Connolly CK, Mamun M, Alcock SM, Prescott RJ. The Darlington and Northallerton prospective asthma study: best function predicts mortality in the first 10 years. Eur Respir J 1998; in press.

16. Agertoft L, Pedersen S. Effects of long-term treatment with an inhaled corticosteroid on growth and pulmonary function in asthmatic children. Respir Med 1994; 88: 373-381.

17. Haahtela T, Jarvenin M, Kava T, et al. Effects of reducing or discontinuing inhaled budesonide in patients with mild asthma. N Engl J Med 1994; 331: 700-705. 